\title{
NON-COMPRESSIVE SLING FOR MALE SUI
}

Prospective evaluations by two European teams support use of the AdVance ${ }^{\mathrm{TM}}$ male sling to relieve post-prostatectomy stress urinary incontinence (SUI). The symptoms of three-quarters of men in whom the self-anchoring polypropylene mesh was implanted improved dramatically. These improvements were sustained in most recipients for at least 1 year.

The first attempt to use slings to cure post-prostatectomy incontinence was made more than half a century ago. High failure and complication rates, however, led to the artificial urinary sphincter becoming the gold standard treatment.

According to Ricarda Bauer, lead author of a new study published in European Urology, negative aspects of the artificial sphincter (mechanical failure, high cost, and the need for manual manipulation by the patient) have led to a resurgence of interest in slings. Minimally invasive placement of these supports is a particularly attractive feature.

Most of the sling systems that have been developed recently have a compressive mode of action. "[These] slings achieve continence by creating obstruction to relieve incontinence, while still allowing micturition," explains Bauer. "The AdVance ${ }^{\mathrm{TM}}$ sling achieves continence [by] repositioning the sphincter region-which has descended [as a result of] prostatectomy - without creating obstruction."

Good residual sphincter function is essential to the success of this sling procedure. Bauer and colleagues used a 'repositioning test' to assess sphincter contraction. Passive closure with contraction of the entire sphincter circumference upon elevation of the perineum, visualized urethroscopically, was the benchmark. "It is important to consider that the AdVance ${ }^{\mathrm{TM}}$ sling does not work in patients with intrinsic sphincter deficiency," reiterates Bauer.

A retrourethral transobturator approach was used to implant slings in 124 men who passed the repositioning test. Preoperative severity of SUI ranged from mild to severe. Prior conservative therapy had not provided symptomatic relief. After 6 months of prospective follow-up, more than $80 \%$ of recipients reported markedly better urinary control. These improvements were sustained at 1 year $(n=70)$.

"Even in the case of 'failure' (that is, no improvement of symptoms) [patients'] urinary conditions remain, at worst, unchanged." So states Jean-Nicolas Cornu, lead author of a second report on the mid-term outcomes of AdVance ${ }^{\mathrm{TM}}$ sling placement. His team followed 102 men for a median of 13 months, and also noted a durable $80 \%$ success rate.

The procedure was notably less effective among the small subsample of patients who had received radiation therapy for prostate cancer (59\%, $n=17$ ). Bauer's team agrees that previous pelvic irradiation is probably an important predictor of outcome. "Based on our experience, only $20 \%$ of patients [who have undergone] radical prostatectomy and additional radiotherapy are eligible for implantation of the AdVance ${ }^{\mathrm{TM}}$ sling."

Both authors stress the importance of adequate surgeon training. According to Cornu, "some key points concerning the dissection of the urethra and positioning of the sling are ... crucial for success."

Suzanne J. Farley

Original articles Bauer, R. M. et al. Prospective evaluation of the functional sling suspension for male postprostatectomy stress urinary incontinence: results after 1 year. Eur. Urol. doi:10.1016/ j.eururo.2009.07.028

Cornu, J.-N. et al. The AdVance transobturator male sling for postprostatectomy incontinence: clinical results of a prospective evaluation after a minimum follow-up of 6 months. Eur. Urol. doi:10.1016/j.eururo.2009.09.015 